\title{
LE VOCABLE « SAHEL 》 COMME INDICATEUR DISCURSIF DE L'ESPACE IDENTITAIRE DU NORD-CAMEROUN
}

The term 'sahel' as a discursive indicator for the identity space

of North Cameroon

\section{Zacharie Hatolong Boho \\ École Normale Supérieure de l'Université de Maroua, FUID/IUDI de Mokolo, Cameroun}

RÉSUMÉ : Le langage ou les pratiques langagières sont susceptibles de créer des lieux communs où se cristallisent, symboliquement ou concrètement, les identités culturelles. La mise en mots de l'espace sahélien du Cameroun à travers le vocable « Sahel » et ses dérivés terminologiques en est une illustration. En effet, le mot sert de descripteur et participe d'une géographie tranquille de la socio-culture ou d'une sémiotique du quotidien. Si « Sahel » est le mot désignateur d'un vaste univers socioculturel, « sahélitude » en est la pratique, c'est-à-dire le fondement d'un modèle de discours identitaire ou d'une vision politique objective. Les discours sur fond de déchirement et d'exclusion sociale s'étant montrés inopérants pour les sociétés en quête d'intégration et d'une politique publique plus juste, les positionnements relativistes et consensuels s'imposent comme mesure palliative. L'éconyme peut donc supplanter l'anthropo-socionyme pour constituer un mode in vivo de dire réflexif ou de dire altéritaire.

Mots CLÉs : sahel, sahélitude, indicateur identitaire, socio-culture, praxis langagière

RESUMEN: El lenguaje o las prácticas lingüísticas son susceptibles de crear lazos comunes en los que cristalicen, simbólicamente o específicamente, las identidades culturales. La plasmación del espacio saheliano de Camerún mediante el vocablo «Sahel»y sus derivados terminológicos son un ejemplo de ello. De hecho, esta palabra describe y participa en una geografía tranquila 
de la sociocultura o en una semiótica del día a día. «Sahel» es el término que designa un universo sociocultural vasto y la «sahélitude» es su práctica, es decir, los cimientos de un modelo de discurso identitario o de una visión política objetiva. Dado que los discursos centrados en el desgarro y la exclusión social son inoperativos para las sociedades que buscan la integración y políticas públicas más justas, los posicionamientos relativistas y consensuales se imponen como medida paliativa. Por tanto, el ecónimo puede suplantar al antroposociónimo para constituir un modo in vivo de decir reflexivo o alteritario.

Palabras clave: sahel, sahélitude, indicador de identidad, sociocultura, praxis lingüística.

RESUM: El llenguatge o les pràctiques lingüístiques són susceptibles de crear lligams comuns en què es materialitzen, simbòlicament o específicament, les identitats culturals. La transmissió de l'espai sahelià de Camerun amb el vocable «Sahel» $\mathrm{i}$ els seus derivats terminològics en són un exemple. De fet, aquesta paraula descriu i participa a una geografia tranquil·la de la sociocultura o a una semiòtica de la vida diària. «Sahel» és el terme que designa un univers sociocultural vast i la «sahélitude» és la seua pràctica, és a dir, els fonaments d'un model de discurs identitari o d'una visió política objectiva. Com que els discursos que giren al voltant de l'aillament i l'exclusió social són inoperatius per a les societats que busquen la integració i polítiques públiques més justes, els posicionaments relativistes i consensuals s'imposen com a mesura pal-liativa. Per tant, l'ecònim pot substituir l'antroposociònim per a constituir un mode in vivo de dir reflexiu o dir alteritari.

Paraules clau: sahel, sahélitude, indicador d'identitat, sociocultura, praxi lingüística.

ABSTRACT: Language and linguistic practices can create common places where cultural identities are symbolically or concretely crystallized. An illustration of this is the use of the term 'Sahel' and its terminological derivatives to refer to the Cameroonian Sahel space. Indeed, the word describes and contributes to a quiet socio-cultural geography, or to everyday semiotics. 'Sahel' 
is the word used to designate a wide socio-cultural universe, and 'sahélitude' is its practice, that is, the basis for an identity discourse model or an objective political vision. Since discourses based on discordance and exclusion are unworkable in societies seeking integration and fair public policy, relativist and consensual positioning are imposed as a palliative measure. Consequently, the econym can supplant the anthropo-socionym to constitute an in vivo way of saying reflexive or alterity.

KEYWORDs: sahel, sahélitude, identity marker, socio-culture, language praxis.

\section{Introduction}

Les discours scientifiques, politiques ou idéologiques en disent souvent long sur le sentiment d'appartenance à un espace identitaire, à des enjeux et défis communs. Beaucoup de chercheurs ont démontré l'homologie existant entre les pratiques socio-langagières ou discursives et l'expression ou la représentation identitaires (Charaudeau, 2001, 2002, 2009 ; Boudreau, 2001 ; Vezeanu, 2004, etc.). Le concept d'ethos discursif ${ }^{1}$ est indiqué pour rendre compte de « la représentation de soi d'un sujet parlant ou pensant [individuel et collectif] dans son discours »(Duman, 2012 : 191). Dès lors, quels que soient ses fondements premiers et ses ultérieures orientations, le discours à la kirditude $^{2}$ en est un exemple assez révélateur. Au-delà du phénomène d'auto-désignation et d'auto-identification que le narrateur déploie, Kirdi est mon $n m^{3}$ de Jean-Baptiste Baskouda est un discours épique, l'épopée même d'un peuple mosaïqué qui s'étend des troubles frontières Centre-Est-Adamaoua au Cameroun jusqu'aux limites indécises du lac Tchad. Son élément constructeur

1. Les concepts d'ethos et de pathos interviennent de manière récurrente en analyse du discours.

2. Selon le socio-politiste et grand théoricien de la question « kirdi » Alawadi Zelao (2012), " la kirditude exprime la dynamique de rapport entre les communautés indigènes au Nord-Cameroun dans sa phase précoloniale, coloniale et postcoloniale ». Il faut ajouter que cette dynamique de rapport s'est toujours opérée à la fois sous forme d'auto-affirmation et de revendication vis-à-vis des islamo-peuls et de l'État camerounais.

3. Titre d'un récit épique au confluent de la prose et de la poésie, publié en 1993 par Jean-Baptiste Baskouda. 
est un sujet culturel (Cros, 1997), un « je » qui s'identifie à tous les peuples dits kirdis ${ }^{4}$ qui disent haut leur appartenance à un même espace sociologique. Cependant, on pourrait à raison traiter cet argumentaire prétendument fédérateur de partisan, réducteur ou exclusif. L'on cherchera alors à n'accorder de prix qu'aux discours inclusifs et plus conciliateurs. En revanche, peut-on voir dans le fameux « Mémorandum du grand-nord $»^{5}$ du Cameroun ou dans la mobilisation autour de l'Université de Maroua un discours épi-nordiste à large spectre et à caractère régional ?

Voilà, en filigrane, la problématique et le contexte dans lesquels s'inscrit cette réflexion qui s'édifie sur un vocable. Nous voyons dans le mot «Sahel »-qui est plus utilisé dans le paysage graphique du Cameroun septentrional que dans celui de la partie sud - une autre catégorie de « je » discursif susceptible de dire tout le Cameroun septentrional. Nous avons identifié cette expression dans ses occurrences les plus diverses dans les principales villes du Nord-Cameroun, notamment à Ngaoundéré, Garoua et Maroua et en avons constitué un corpus dont l'analyse interdisciplinaire nous permettra d'en déduire quelques pratiques et discours identitaires. Il en découle que l'objectif de l'étude est d'analyser la mise en discours, puis en espace, d'une identité socioculturelle traduite de manière langagière.

Le vocable « Sahel » (ainsi que ses formes dérivées « sahélien/ne ») est utilisé dans divers contextes, différents secteurs ou domaines d'activité : le transport, le commerce, l'habitat, la gastronomie, la presse, les TIC, l'agropastoral, l'esthétique corporelle et vestimentaire, les finances, les associations, les loisirs, etc. Tout le septentrion en est marqué (les données collectées dans les trois capitales régionales n'en constituent qu'un spécimen) tantôt sur les façades et devantures des institutions socio-économico-culturelles, tantôt sur des objets. Sans doute, il s'agit d'une « évaluation sociale des pratiques langagières d'autrui comme de soi-même » (Bulot, 2001), d'une " septentrionalisation » des pratiques et aspirations locales et, surtout, d'une revendication d'appartenance

4. Dans son acception première, « Kirdi » désigne l'ensemble des peuples autochtones et non musulmans du Nord-Cameroun.

5. Une sorte de revendication ethno-régionale, formulée en 2002 par l'élite du Nord-Cameroun à l'intention de l'État camerounais et dont l'objectif était de décrier les déséquilibres et les injustices socio-politiques dont la partie septentrionale est victime. 
à un même espace identitaire. Cependant, ce discours n'est pas niveleur ; l'un et le multiple s'y reconnaissent, comme le souhaiteraient si bien les adeptes de l'intégration nationale ou de la fameuse mondialisation. Par ailleurs, ce n'est pas tant l'acte du « dire » ou du « nommer » qui construit la réalité examinée ; c'est de la coïncidence diversifiante des sujets dans la désignation ou nomination et dans le discours que surgit l'univers sahélien co-construit.

Le recueil des données analysées s'est effectué à partir de l'observation directe, ce qui fait appel à l'ethnométhodologie dont Garfinkel nous dit que :

Les études ethnométhodologiques analysent les activités quotidiennes des membres comme des méthodes qui rendent ces mêmes activités visiblement rationnelles et rapportables à toutes fins pratiques, c'est-à-dire descriptibles (accountable), en tant qu'organisation ordinaire des activités de tous les jours. La réflexivité de ce phénomène est un trait singulier des actions pratiques, des circonstances pratiques, de la connaissance commune des structures sociales et du raisonnement sociologique pratique [...] (Reproduit dans Lallement, 2012 : 230).

Il s'en suit qu'on peut rendre compte - et c'est le cas dans cette étude - de ces accomplissements et raisonnements pratiques au moyen d'une démarche ethnographique sans qu'il y ait besoin d'hypothèses préalables au travail de terrain ${ }^{6}$.

\section{Stratégies dénominatives}

La dénomination et ses stratégies de déploiement renvoient à un ensemble de procédés morphosyntaxiques, lexicaux et stylistiques qui concourent à rendre possible un acte de dénomination et/ou de désignation.

6. Selon Abderrahim Kenaïssi, « pour accéder au sens des pratiques sociales, il suffit d'observer deux principes. D'abord, les considérer toutes, et de plein droit, les plus massives et les plus marginales, comme également significatives de la production de la vie sociale. Du coup, les situations les plus banales, voire anecdotiques, deviennent objets d'étude : une conversation téléphonique, des piétons qui traversent, un élève qui répond ou non à une question, une gamine qui fugue, un transsexuel qui change de sexe... Ensuite, être attentif aux phénomènes tels que décrits par les acteurs, avec une « indifférence ethnométhodologique » qui signifie regard affranchi des hypothèses, des jugements de valeur et autres considérations a priori. Car le fait que les membres soient dotés d'une connaissance familière et d'une compétence analytique confère à leurs pratiques trois propriétés qui les rendent accessibles à la compréhension du chercheur : l'indexicalité, la réflexivité, la descriptibilité »". 
Bien des chercheurs se sont penchés dessus en études linguistiques en général ou en analyses du discours, notamment Kleiber (1974), Cislara et al. (2007), Fèvre-Pernet (2008, 2011), Lecolle et al. (2009). C'est fort de ces travaux que cette réflexion est déployée, s'inscrivant dans le vaste et riche domaine de l'onomastique, se fondant plus précisément sur la désignation des lieux de ville (Bulot, 2004) et des faits urbains qui cristallisent les imaginaires socio-spatiaux des individus d'une sphère géographique bien définie. Avant d'en déterminer la finalité par interprétation, force est d'examiner la ou les manières dont les sujets procèdent à la mise en mots de l'espace social septentrional à travers le vocable « Sahel ». C'est l'aspect empirique du phénomène observé, qui repose essentiellement sur la question « comment», le « quoi » étant consubstantiellement lié à l'objet de recherche qui a été sommairement présenté en introduction. Pour ce faire, l'analyse dans cette première partie s'intéressera au niveau structurel des données, notamment aux aspects morpho-syntagmatique et sémantique.

Du point de vue de leurs formes et du principe d'agencement de leurs éléments constitutifs, la quasi-totalité des noms soumis à cette étude alternent les catégories du nom et de l'adjectif. Les phénomènes syntaxiques qui concourent à la structuration des noms des faits de ville sont essentiellement la détermination et la constellation. Au sens hjelmslévien, la relation de détermination tient lorsqu'un des termes présuppose l'autre et non l'inverse, c'està-dire une relation syntaxique unilatérale. Le corpus comprend beaucoup de structures - s'il n'en est d'ailleurs constitué à plus de $70 \%$ - ayant cette caractéristique, notamment Eil du Sahel, Crédit du Sahel, Tempête du Sahel, Kossam Sahel, Walde Sahel, Water Sahel, Sahel alimentation, Sahel couture, Sahel shop, etc. Les six derniers exemples, quoique se calquant sur le modèle syntaxique de la langue anglaise, ne diffèrent pas des trois premiers. À y voir de près, tous ces syntagmes rappellent la structure du nom complémenté ou du noyau et de l'expansion, c'est-à-dire la structure X de/du Y.

Quant à la constellation, autre concept emprunté à Hjelmslev, elle caractérise deux éléments qui, malgré la compatibilité qu'ils entretiennent, ne se présupposent pas du point de vue syntaxique. Les cas pour l'illustration sont, entre autres, HOTEL le SAHEL, Complexe Sahel clean pressing, La tribune sahélienne presse, Sahel call Box transfert, Labo Sahel photo, Sahel Springs 
eau minérale naturelle, Sahel Spring, etc. Ici, le mécanisme consiste en une juxtaposition de deux entités autonomes. Ces énoncés sont courants dans la signalétique et les messages publicitaires qui démontrent l'économie du langage et qui rappellent, une fois encore, la syntaxe de l'anglais.

En définitive, quelle que soit sa position dans le syntagme et le rapport entretenu avec le reste, le terme «Sahel » joue un rôle prépondérant, les autres éléments de catégories grammaticales diverses lui servant de déterminants, de descripteurs, de qualificateurs ou d'attributaires. La détermination et la constellation constituent des stratégies dénominatives descriptives, pour une dénomination fonctionnelle (Fèvre-Pernet, 2011). Dans ce sens, toutes les occurrences du corpus sont des schèmes appellatifs (Fèvre-Pernet, 2008) à valeur d'identification. Ainsi, on assiste à des pratiques socio-langagières basées sur une dénomination qui opère des découpages du réel urbain et des praxis sociales.

\section{Sahel : dévoilement de soi à soi et à l'autre}

Le terme « Sahel » comme indicateur de mise en mots de l'espace est le point de départ d'un phénomène consistant en la sémiotisation et la mise en perspective du hic et nunc. C'est une géographie tranquille du quotidien et une pratique socio-spatiale (Guy de Méo, 1999) ; c'est également une anthropologie, sociologie, psychosociale, histoire, etc., du quotidien. Derrière le vocable, il y a des personnes, des cultures et des activités journalières qui sont dites ou représentées. Le dévoilement de soi à soi et à l'autre consiste en un processus d'auto-désignation et de mise en discours de soi. Sans prétention d'exhaustivité, on peut présenter dans le tableau qui suit quelques aspects de cet univers dit et construit :

\begin{tabular}{|c|c|}
\hline Secteurs d'activité & Exemples \\
\hline Transport & Sahel express \\
\hline Habitat & Minicité le Sahel \\
\hline Loisir & SOURIA-BAI Discothèque - Sahel- \\
& Dougoï \\
\hline
\end{tabular}




\begin{tabular}{|c|c|}
\hline Commerce & Alimentation du Sahel \\
\hline $\begin{array}{c}\text { Esthétique corporelle/vesti- } \\
\text { mentaire }\end{array}$ & Haute coiffure du Sahel \\
\hline TIC & Sahel espace informatique \\
\hline Hôtellerie/Restauration & Hôtel le Sahel \\
\hline Agropastoral & PHYTos du Sahel \\
\hline Presse & Eil du Sahel \\
\hline Association & GIC des éleveurs du Sahel \\
\hline Banque/finance & Crédit du Sahel SA \\
\hline Enseignement & Institut Supérieur du Sahel \\
\hline
\end{tabular}

Tableau : Les données selon les domaines de référence.

En se mettant en discours à travers le vocable « Sahel », le Cameroun septentrional s'auto-définit identitairement et se réclame du Sahel en général. Par conséquent, il s'y joue la partition d'une multiple intégration ou, tout simplement, une médiation entre l'ici et l'ailleurs nationaux et/ou transnationaux. En effet, ce terme est un référent englobant à plusieurs titres. Même si l'origine du nom « Sahel » est discutée, bien des auteurs ont fini par convenir de son origine arabe. Ce vocable signifierait « rivage » ou « littoral », pour désigner la frange qui borde le sud du désert du Sahara (Bernus, 1981). Selon Hiernaux et Le Houérou (2006), le terme proviendrait également d'un mot arabe signifiant " rivage ", dans le sens de " rivage sud du Sahara ", soit d'un autre mot arabe signifiant «plaine », « étendue plane » ou vallonnée. La définition nous en est d'ailleurs donnée dans le travail de ces deux derniers auteurs :

Le Sahel est une entité biogéographique définie en première instance par son climat tropical aride à semi-aride, contrôlé par la mousson du golfe de Guinée et l'Harmattan (alizé) saharien. Mais au cours du quaternaire la végétation sahélienne a dû s'adapter à des fluctuations climatiques entre les climats tropicaux humide et aride, voire hyperaride. (Hiernaux et Le Houérou, 2006 : 51). 
Ici, deux choses accrochent. D'une part, le fait que le mot « Sahel» soit repris et emprunté par plusieurs langues est révélateur d'une vision globale $\mathrm{du}$ fait conceptuel et identitaire. Dans le grand-nord du Cameroun, ceux qui utilisent ce vocable pour désigner leurs structures et activités l'auraient emprunté à l'arabe, au français, à l'anglais ou à des langues locales. L'origine du mot importe peu ; l'important est qu'il soit unanimement et symboliquement utilisé pour dire un espace identitaire au confluent de l'ici et de l'ailleurs. « Espace charnière entre la Méditerranée et l'Afrique subsaharienne » (Mehdi Taje, 2010), le Sahel renvoie à une réalité absolument plurielle.

Ainsi, en tant que dénominateur lexical commun, le mot « Sahel» devrait constituer l'entrée liminaire du dictionnaire Le Nord-Cameroun à travers ses mots (Seignobos \& Tourneux, 2002). Son usage dans plusieurs secteurs d'activité fait de ce terme l'un des identifiants à même de mieux dire tout l'univers septentrional. Ce renvoyant lexical pluri-référentiel d'auto-identification est également un médiateur vers l'altérité. Au même titre que beaucoup d'autres termes de caractère néologique (négritude, tigritude, kirditude, merditude, algérianitude, tchaditude, corsitude, etc.), « sahélitude » serait la néologie idoine pour expliquer ce phénomène. C'est peut-être une réaction aux multiples mécanismes d'étiquetage de soi par les autres, mécanismes que peuvent bien illustrer les voix ou clichés réducteurs tels que : wadjo, haousa, mouton du nord, awara, tok tok, musulman, etc. Le cas échéant, on conclurait au type de positionnement identitaire (Kouidri, 2009) qui s'opère chaque fois qu'il y a confrontation entre identité et altérité. Une autre conjecture est envisageable, qui invaliderait la thèse de revendication ou affirmation identitaires pour rendre plausible l'idée de ritualisation du quotidien. Ainsi, sans égard pour l'altérité et sans aucun souci de différentiation, l'expression identitaire se manifesterait aussi naturellement que la palpitation du cœur ou le rythme respiratoire. Sa motivation lui serait intrinsèque et résiderait dans son propre dynamisme de (re)construction, de (re)configuration et d'auto-évaluation.

\section{Sahélitude comme contre-pied des discours « à la kirditude »}

Le discours « à la kirditude » peut, peu ou prou, s'envisager comme une déconstruction ou désagrégation d'une identité antérieurement large. En 
effet, au fil des temps, le concept « kirdi » a désigné une réalité de plus en plus restreinte puisque le vent de l'islamisation, quoique n'étant plus aussi fort qu'aux temps des conquêtes foulbé et au cours du premier régime, continue de balayer les trois régions septentrionales. S'il est vrai que la kirditude trouve sa substance dans la masse démographique constituée des groupes ethniques endogènes du septentrion, cette substance s'est amoindrie chaque fois qu'un seul kirdi s'est converti à l'islam. À propos, et cela pour diverses raisons (accès aux ressources, aspiration aux positions de pouvoir, etc.), le nombre d'individus traités de « kado » (pluriel « habé »), d'impurs, de mécréants ou « vivant une convivialité cosmique régie par un attachement aux esprits surnaturels » (Houli, $2006: 84$ ) a décru au fil des ans. Dans la logique ou la dialectique peuhl-kirdi, il peut s'observer une dékirdisation en faveur de l'islamisation, processus dont l'importance réside tant dans la variable conceptuelle que dans la réalité sociodémographique. Aux antipodes donc d'un discours identitaire purement réducteur se situera une vision consensuelle ou fédératrice.

Déjà, la question même de l'existence d'une identité nordiste se pose avec pertinence et l'on se rendra compte que le critère anthropo-socio-centré se sera avéré limitant. En effet, fût-ce de l'intérieur ou de l'extérieur, comment le nordiste est-il défini et envisagé ? Si l'identité va de pair avec la prise de conscience de soi (Charaudeau, 2009), qu'est-ce qui permet aux nordistes de prendre conscience d'être ce qu'ils sont ? De l'intérieur, les traits antinomiques comme autochtones/allogènes, montagnards/habitants des plaines, sédentaires/nomades, agropasteurs/commerçants, musulmans/non-musulmans, etc., peuvent caractériser les peuples du Nord-Cameroun. Ces considérations réfèrent bel et bien aux différentes facettes et dynamiques identitaires auto-évaluatives du septentrion. Cependant, il faudra également mentionner ses différentes " imbrications culturelles qui tiennent lieu d'identité » (Duchêne, 2006 : 193). La pluralité des souches anthropo-sociologiques, la diversité linguistique et des pratiques artistico-culturelles, etc., définissent le septentrion tout autant que le reste du pays.

Par ailleurs, le nord se conçoit en tant que tel parce qu'il s'inscrit dans une logique d'opposition ou de comparaison avec le sud. De ce point de vue, le grand-nord serait cette partie du pays prétendument caractérisée 
par la sécheresse, la pauvreté, la sous-scolarisation, etc. Bien évidemment, même s'il y a une part de vrai dans ces caractérisations, il n'en demeure pas moins qu'elles sont révélatrices des mécanismes de mise à distance (Mulo Farenkia, 2011) et des considérations stéréotypées. Les appellations telles que « Hausa », « Wadjo », « Moutons du nord », « Alhadji », etc., réitérons-le, en disent long sur le phénomène de mise en discours de l'autre, sur l'étiquetage des peuples du septentrion ${ }^{7}$. Cependant, on se rendra vite compte du caractère réducteur de ces attributs relativistes dont l'ancrage tient à l'habitat, à l'origine sociologique, à l'activité socio-économique ou à la religion. Ces déterminants à prégnance socioculturelle ne sont pas l'apanage des seuls peuples du septentrion, pas plus d'ailleurs que des caractéristiques telles que la pauvreté ou la sous-scolarisation.

Pour mieux « identifier » le Cameroun septentrional, il faudrait appliquer des marqueurs absolus ou globalisants, des déterminants qui incluraient des dimensions autant monolithiques que plurielles. L'optique n'est ni d'évacuer les représentations univoques ou monocentrées des sociocultures en présence, ni d'homogénéiser les diverses dynamiques du social. L'intention n'est pas non plus de condamner purement et simplement des discours « à la kirditude » (la Kirditude étant un mouvement né dans un contexte sociopolitique bien précis et ayant eu sa raison d'être), mais de démontrer les limites de certains artifices identitaires. En définitive, il conviendrait de réfuter l'ethnicisation simpliste ${ }^{8}$ des points de vue et énoncés consistant à dire tout le Cameroun septentrional. Dans cette quête conceptuelle, il faudra trouver au Cameroun septentrional une ou quelques identités remarquables. Tâche bien difficile, étant donné que cette partie du pays se meut dans une logique « qu'impose un monde qui est en soi un univers pluriel» (Dussouy, 2010). Tâche difficile

7. Vu du septentrion ou d'ailleurs, le Sud-Cameroun peut tout autant être l'objet de ces qualifications stéréotypantes, ce qui relancerait le débat quant à l'objectivité du processus d'identification.

8. Dans un livre récemment publié (2011), Paul Abouna parle de l'ethnicisation du pouvoir et des pratiques. Il aborde là un ensemble de pratiques réelles dans l'environnement sociopolitique du Cameroun en particulier et de bien des pays en Afrique. Cependant, lorsqu'il mentionne L'Eil du Sahel comme un exemple d'ethnicisation de la presse, son analyse manque de pertinence car le septentrion que couvre prioritairement cet organe de presse ne saurait se réduire à une entité ethnique. 
également d'autant plus que les temps présents, c'est-à-dire la « conjoncture postmoderne » (Trépos, 1998) ou le « moment postmoderne » (Chivallon, 2004), font de la socio-culture une notion complexe et insaisissable. Si les discours socio-idéologiques ou les forces socioculturelles, notamment les discours « à la kirditude », les motions ou énoncés mémorandaires unilatéraux, les groupes sociaux ou ethniques, etc., n'ont pas acquis la notoriété suffisante pour définir le septentrion, il faudra adopter un autre critère définitionnel. Pour cela, l'on n'ira pas explorer les nuages ; il existe dans les appellations et les discours actuels des traits de caractérisation susceptibles de faire sens et consensus.

Les dénominations telles que le « nord », le « Nord-Cameroun », le « grand-nord », le « septentrion », etc., ainsi que leurs variantes adjectivales (« nordiste » ou « septentrional ») nous paraissent plus consensuelles - à plus d'un égard - que certains référents identitaires simplificateurs. D'une part, le « nord » ou le « septentrion » représentent le « lieu commun» physique qui comprend l'Adamaoua, le nord et l'extrême nord, constituant ipso facto l'une des deux composantes géographiques du Cameroun. Envisager le Nord-Cameroun en tant qu'espace physique et en comparaison du Sud-Cameroun est d'autant plus pertinent que, non seulement une identité se conçoit mieux sur la base du " principe d'altérité » (Charaudeau, 2009), mais la confrontation de ces deux espaces est assez révélatrice dans les pratiques et les imaginaires aussi bien des Camerounais que des étrangers. Sur le plan politique, le paradigme nord-sud est une formule de gestion et de distribution des positions de pouvoir entre des blocs ethno-régionaux. D'autre part, l'espace en tant que tel n'est ni indifférent, ni un no man's land; il cristallise des réalités anthropo-socio-culturelles et des réalités identitaires fortes. Dans le cas d'espèce, il suffit très souvent $d$ 'articuler le terme « nordiste » pour qu'un type d'homme et de femme soit nommé et identifié par rapport à un certain nombre de déterminants socioculturels. Il faut, dans ce processus d'identification, privilégier les données ratifiées par le temps - sans pour autant oublier les erreurs de l'histoire - telles que le passé commun, la géographie, les emblèmes, la patrie et les aspirations convergentes.

C'est dans une vision écocentrée ou écosystémique de caractérisation que s'inscrit la présente démarche épistémologique. Bien sûr, cette démarche 
se veut relative dans la mesure où il serait embarrassant d'envisager une identité humaine qui ne soit pas basée sur la vision anthropomorphique. En fait, l'éco-vision manquerait de pertinence si elle se concevait en tant qu'unique figure de définition du Cameroun septentrional ; tout comme la seule anthropo/ socio-vision perdrait en crédibilité épistémologique. Cependant, la détermination de l'espace identitaire à partir d'un trait naturel, le Sahel dans ce cas de figure, nous semble le seul dénominateur commun, surtout qu'il est ratifié par une pratique discursive collective. Sans pour autant relancer le vieux débat opposant la nature à la culture, il convient de souligner l'incontournable interaction constante entre le milieu naturel et le milieu humain. D'aucuns parlent d'ailleurs de déterminisme ${ }^{9}$ pour appréhender l'incidence de certains facteurs naturels sur les réalités anthropo/socio-culturelles. Dans le cas du Cameroun septentrional, force est de noter que bien des dynamiques (sociales, économiques, etc.) sont déterminées ou conditionnées par les variables naturelles. De l'Adamaoua à l'extrême nord, les dynamiques des populations, la gestion $\mathrm{du}$ foncier, les activités socio-culturelles, économiques, agropastorales, etc., sont fonction de l'environnement ou de l'espace mis en mots et en discours à partir du concept « Sahel ». Cette réalité physique présente à la fois plusieurs facettes : espace de vie, espace vécu, espace social(isé), territoire, espacetemps, espace exploité, espace privé, espace public, lieux de sémiotisation, lieux de représentation, structure, système, etc. Tout ceci peut être repris par Guy Di Méo (1999 : 90) :

La rencontre historique (datée et interactive) de groupes humains et de milieux géographiques particuliers produit des civilisations originales. Chacune, ou presque, donne naissance à des univers techniques et à des schèmes d'organisation du travail spécifiques. Ces sociétés, en prise à la fois concrète et idéologique avec leurs espaces, dessinent des géographies objectives qui témoignent de leur territorialisation. Sur la base de cette objectivation territoriale et du langage symbolique des structures cognitives (incorporées par chaque sujet) qui lui donne corps, des liens étroits rapprochent les innombrables itinéraires personnalisés du quotidien, les pratiques spatiales et les rapports sociaux qui les accompagnent, les contingences qui les contraignent, les initiatives individuelles qui les infléchissent et les représentations de tous ordres qui les modèlent.

9. Évidemment, il ne s'agit pas ici du déterminisme classique pur et dur. 
C'est, au demeurant, en vertu de ces considérations socio-spatiales que des disciplines telles que l'anthropologie, la sociologie, la psychologie, etc., de l'environnement ou de l'espace se sont développées. Vu son caractère fort complexe, le problème qu'explore la présente réflexion se soumettrait avantageusement aux rayons lumineux d'un faisceau interdisciplinaire aux paradigmes théoriques et conceptuels opératoires.

\section{Vers un discours intégrationniste : combiner réductionnisme et intégrationnisme}

Depuis son accession à l'indépendance en 1960, le Cameroun cherche désespérément la voie de l'intégration nationale. Il y a eu, du point de vue onomastique, une tentative de nommer par des mots non discriminants les lieux ou les institutions communs de la jeune nation. Systématiquement, les divisions et subdivisions administratives se sont vu attribuer des noms au référent géographique ou écologique. C'est le cas de la majorité des régions dont les noms renvoient fondamentalement aux quatre points cardinaux : le nord, le sud, l'ouest, l'est, l'extrême nord, le nord-ouest et le sud-ouest. Le centre et le littoral sont également des renvoyants géographiques qui obéissent à la même logique écocentrée. Seul l'Adamaoua ${ }^{10}$ s'inscrit dans le principe de « pipolisation » (Engel, 2012), non pas pour ratifier la contingence mais pour en référer à la mémoire collective. Ce dernier principe de dénomination relève de ce que Paveau (2008 : 23-24) décrit en ces termes :

Dans une perspective discursive qui privilégie la mémoire et la cognition socio-culturelle1, le toponyme n'accomplit pas seulement une dénomination géographique, mais dessine des cheminements sémantiques complexes, contingents et parfois originaux, à travers les cadres culturels, identitaires, affec-

10. Ce terme est répertorié et expliqué dans le dictionnaire de Christian Seignobos et Henry Tourneux (2002: 12) : < fulfulde [Aadamaawa] < [Aadama], nom de personne, d'origine arabe. (1) province historique de l'empire peul de Sokkoto ; (2) nom d'un groupe de langues de la famille Niger-Congo. Cette vaste province de l'empire peul de Sokkoto, pratiquement incluse dans le nord du Cameroun, tire son nom de son organisateur, Modibo Adama Hasana [modibbo Adama Hasana]. Nommé émir du Sud [amiru fombina] par le shéhou de Sokkoto, Modibo Adama administrait une poussière d'États qui allaient des yaérés au nord, avec les principautés de Petté [pette] et de Bogo jusqu'aux forêts tropicales, au sud, avec celles de Banyo [Bamnyo] et de Tibati [Tibati]. Yola [Yola], la capitale, située sur les rives de la Bénoué, fut fondée en 1829. 
tifs et mémoriels d'un sujet ou d'un groupe. Au sein d'une approche des faits langagiers et discursifs qui articule discours et cognition, le toponyme (comme tout nom propre d'ailleurs) peut être envisagé comme un lieu de mémoire discursive et un organisateur socio-cognitif permettant aux locuteurs de construire une histoire collective. Il tient en effet une place importante parmi les éléments qui constituent des lignées discursives, entendues comme des configurations sémantiques transmises par les locuteurs-prédécesseurs à partir de cadres prédiscursifs collectifs (Paveau, 2006). Le toponyme est un des « agents de transmission » qui inscrivent dans les discours le fil de ces lignées.

La forte tendance à éviter les anthroponymes et socionymes dans l'onomastique institutionnelle et officielle au Cameroun trouve également son illustration dans les noms des circonscriptions administratives (départements et arrondissements), des locomotives et des avions de ligne. Généralement, ce sont les noms de cours d'eau ou de reliefs qui sont donnés, à l'instar du Nyong, du Dja, de la Bénoué, du Mayo-Sava, du Mayo-Tsanaga, de la Sanaga, du Wouri, du Mungo, du Mfoundi, etc. Non moins illustratifs sont les noms d'institution pour lesquels on utilise la particule « Cam-» (Cameroun/ Cameroon) comme préfixe ou suffixe : Régifercam (devenu Camrail), Camair (devenu Camairco), Sopecam, etc.

L'option pour une onomastique ou une auto-désignation dépersonnalisée et désocialisée, rappelons-le, est révélatrice d'une idéologie politique qui visait à placer le Cameroun sur la voie de l'intégration nationale. Cependant, l'acte discursif et symbolique de dénomination se heurte à la réalité des clivages historiques ou séculaires et de la diversité socioculturelle. En effet, entre autres passifs coloniaux, l'équation des deux Cameroun a toujours constitué un véritable casse-tête, autant pour les dirigeants que pour les citoyens. Or, il n'y a pas que la situation bipolaire de la nation qui fait problème ; il y a sa diversité socioculturelle et linguistique qui en rajoute au caractère complexe. Des politiques, des pratiques et des discours s'enchâssent ou se succèdent dans le temps, mais ne trouvent pas la voie idéale devant aboutir à la fameuse intégration nationale. Au-delà de la possibilité intelligente de se poser la question de savoir ce que signifie cette intégration tant recherchée, ou si elle 
existe vraiment, nous choisissons délibérément de diagnostiquer les pratiques en cours afin de proposer une des mille et une solutions.

Par ailleurs, on peut, à partir de la tendance écosystémique qui soustend la toponymie au Cameroun, émettre l'hypothèse d'une fuite en avant/ de responsabilité. En effet, la prise en compte quasi exclusive des référents naturels dans l'acte dénominatif ou l'onomastique nationale semble inscrire l'ingénierie sociopolitique du Cameroun dans une situation phobogène. Comme déterminée par une névrose phobique, il se développe une phobie du social et de l'humain au profit de l'écologique. Cette idéologie prétendument neutraliste qui peut friser l'escapisme (Giannetti, 2002) se manifeste également à travers la politique linguistique : érection de deux langues coloniales - le français et l'anglais - en langues officielles et assignation du statut symbolique aux langues et cultures nationales. Phobie du social ou de l'humain ? Tout laisse, du moins, conclure à une démarche utopique car, comme le postule Mannheim (1929 : 64), « un état dresprit est utopique, quand il est en désaccord avec liétat de réalité dans lequel il se produit».

Conscients du caractère pluriel des sociocultures au Cameroun, les politiques ont contradictoirement opté pour la méthode intégrationniste ou maximaliste qui consiste à envisager la nation comme un seul bloc monolithique ou à la fragmenter en grands ensembles socioculturels gouvernables. Les antagonismes préexistants sont alors ratifiés, les autres dichotomies sont créées : Cameroun anglophone vs Cameroun francophone, Grand Sud vs Grand Nord, etc. C'est la politique de l'autruche ou de l'occultation qui, sur le plan glottopolitique par exemple, a abouti au fameux bilinguisme officiel ou institutionnel. C'est-à-dire, au nom d'une intégration nationale, qu'on promeut à outrance les langues officielles et étrangères au détriment des langues locales ${ }^{11}$ auxquelles on réserve une place symbolique et patrimoniale. La perspective réductionniste ou minimaliste, sans pour autant s'ériger en modèle satisfaisant, aurait été efficace dans la mesure où la reconnaissance et la promotion des minorités concourent forcément à l'édification d'une conscience nationale portée vers l'esprit de groupe ou de nation. Le contraire

11. De nos jours, six langues étrangères (l'allemand, l'arabe, le chinois, l'espagnol, l'italien et le portugais) sont en compétition dans le sous-système éducatif francophone alors qu'aucune langue nationale n'est à même de bénéficier de ces statut et fonction. 
engendre les injustices ou inégalités et laisse inexorablement du feu sous la cendre.

Ainsi, ni le minimalisme ni le maximalisme ${ }^{12}$ ne sont efficaces comme stratégies de gouvernance et de quête d'intégration. On proposerait alors le modèle minima-maximaliste qui combinerait les deux modèles individuellement inopérants. C'est le propre de la théorie gestalt, qui permet à la fois d'avoir une vision panoramique et d'avoir pleine conscience de l'existence non accidentelle des particularités. C'est aussi la leçon du disciple Martinet au maître Saussure ou le complément pertinent qu'apporte le fonctionnalisme au structuralisme. Il ne suffit plus de considérer la société ou la nation comme un système amorphe, mais d'avoir conscience d'une dynamique interne, une dynamique impulsée du bas. La difficulté du modèle ambiant réside dans les politiques in vitro, les politiques de laboratoire façonnées par le haut qui consistent à tout penser à la place des citoyens pris pour des automates. Or, le mécanisme discursif qui concourt à la sahélitude tient à une action in vivo, c'est-à-dire à une conscience d'appartenir, malgré les différences, à un grand ensemble socioculturel. Cette perspective revêt une importance assez grande dans la mesure où, si tout nordiste reconnaît appartenir à tout le septentrion et non pas exclusivement à sa propre région (l'Adamaoua, l'extrême nord ou le nord), il est capable d'exprimer son appartenance à la nation camerounaise.

\section{Conclusion}

$\mathrm{Au}$ terme de ce parcours consistant à explorer les potentialités discursives d'une praxis langagière basée sur la désignation, il convient de conclure que toute la texture socioculturelle du Nord-Cameroun a été

12. Pour Warren Motte, le minimalisme se manifeste ainsi: "It's an art that insists upon reduction and mobilizes it as a constructive principle ». Cependant, Warren Motte affirme que la notion de réduction se joint à celle de l'amplification : "Through a reduction of means, minimalists hope to achieve an amplification of effect " " (Myrthe Holvoet, $2013: 8$ ). S'opposant substantiellement au concept antérieur, le maximalisme renvoie à la perspective ensembliste des choses. Les deux concepts peuvent bien cristalliser le faux jeu métonymique : la partie pour le tout et le tout pour le tout. 
nommée/construite à partir du vocable «Sahel » et de ses dérivés terminologiques. Du point de vue strictement référentiel, le vocable en question désigne une réalité socio-spatiale polymorphe qui, socio-culturellement parlant, constitue une transition entre les parties nord et sud de l'Afrique. Double métonymie : le Sahel camerounais est une partie intégrante du territoire national, en même temps qu'il se projette jusqu'aux berges de la Méditerranée. D'autre part, la référence faite est consubstantielle au fait que le Cameroun est une Afrique en miniature. Au plan identitaire, « Sahel » est le mot par excellence du Cameroun septentrional dans la mesure où il peut être pris comme un dénominateur commun référentiel d'un univers qui cristallise à la fois le même et l'autre. La sahélitude qui en découle peut alors, en tant que modèle discursif, constituer le contre-pied d'une vision « à la kirditude » construite sur fond de conflictualité et d'exclusion de l'autre. En tant que déterminant écosystémique, la sahélitude s'envisage comme un modèle consensuel, restituant ainsi les idéaux intégrationnistes que l'État-nation du Cameroun construisait déjà sur la base d'une onomastique écocentrée. Ceci d'autant plus que « la plupart des identités affichent une composante géographique, une spatialité qui les renforce et les rend plus prégnantes. Elles s'expriment donc, souvent, par ces médiations du social et du spatial que forment les lieux, les territoires, les paysages... » (Di Méo, 2007 : 69).

Le discours-alternative de la sahélitude stipule que la reconnaissance par une partie - non homogène - de son appartenance à un tout national ou culturel est le point de départ d'un processus efficient susceptible d'aboutir à l'intégration que recherchent les sociétés plurielles. Pour ce qui est du Cameroun, une telle vision guiderait mieux dans la conception des politiques publiques qui sont très souvent entravées par le repli identitaire et les cris des minorités. Le modèle minima-maximaliste qui est ici prôné tient compte à la fois des particularités culturelles et de la globalité, et sert de projet intégratif pour une société plus réaliste et plus juste. 


\section{Références}

Aвouna, P. (2011) : Le pouvoir de l'ethnie - Introduction à l'ethnocratie, Paris. L'Harmattan.

Alawadi, Z. (2012) : « Système des Nations Unies de protection et de promotion des droits des minorités : l'exemple des minorités Montagnardes à l'Extrême-Nord Cameroun », communication présentée à l'Atelier national sur : «La participation des minorités aux prochaines élections municipales et législatives au Cameroun », organisé par Minority Rights Group, Yaoundé, 28-29 septembre.

Bernus, E. (1981) : Touaregs nigériens. Unité culturelle et diversité régionale d'un peuple pasteur. Paris, Ed. de l'Office de la recherche scientifique et technique Outre-Mer.

Boudreau, A. (2001) : « « Langue(s), discours et identité » », Francophonies d'Amérique, $\mathrm{n}^{\circ}$ 12, 93-104.

Bulot, T. (2001) : «L'essence sociolinguistique des territoires urbains : un aménagement linguistique de la ville ? », Cahiers de sociolinguistique, $\mathrm{n}^{\circ} 6,5-11$.

—(dir.) (2004) : Lieux de ville et identité (Perspectives en sociolinguistique urbaine, vol 1, Paris, L'Harmattan.

Charaudeau, P. (2001) : "Langue, discours et identité culturelle », Revue de didactologie des langues-cultures 2001/3-4, $\mathrm{n}^{\circ} 123$, pp. 341-348.

- (2002) : " L'identité culturelle entre langue et discours », Revue de l'AQEFLS vol. 24, $\mathrm{n}^{\circ} 1$, consulté le 12 septembre 2016, URL: http://www.patrick-charaudeau.com/L-identite-culturelle-entre-langue.html

- (2009) : « Identité sociale et identité discursive. Un jeu de miroir fondateur de l'activité langagière "), in P. Charaudeau (dir.), Identités sociales et discursives du sujet parlant, L'Harmattan, Paris, 2009, consulté le 12 septembre 2016, URL: http://www.patrick-charaudeau.com/Identite-sociale-et-identite, $217 . \mathrm{html}$

Charmes, E. (2005) : La vie périurbaine face à la menace des gated communities, L'Harmattan, Paris.

Chivallon, C. (2004) : « Débattre autour du postmodernisme : commentaires de textes choisis », L'Espace géographique, n 1, pp. 43-58.

Cislaru, G. $\boldsymbol{E T} \boldsymbol{A} \boldsymbol{A}$. (eds.) (2007) : L'acte de nommer. Une dynamique entre langue et discours, Presses Sorbonne Nouvelle, Paris. 
Cros, E. (1997): El sujeto cultural: sociocrítica y psicoanálisis, Ediciones Corregidor, Buenos Aires.

Di Meo, G. (2007) : « Identités et territoires : des rapports accentués en milieu urbain? ", Métropoles, $\mathrm{n}^{\circ}$ 1, 68-94.

Dorier-Apprill E. \& Van Den Avenne, C. (2002) : « Usages toponymiques et pratiques de l'espace urbain à Mopti (Mali) - La toponymie entre linguistique et géographie », Marges linguistiques, $\mathrm{n}^{\circ} 3,151-158$.

DuChene, N. (2006) : " Littérature créole et stratégies identitaires », CAUCE, Revista Internacional de Filología y su Didáctica, no 29, 191-206.

Duman D. C. (2012) : "L'identité et ses représentations : Ethos et Pathos », Synergies Turquie, $\mathrm{n}^{\circ}$ 5, 187-200.

Dussouy, G. (2010) : « Pragmatisme et géopolitique - Les opportunités méthodologiques d'une retrouvaille épistémologique. ", L'Espace Politique [En ligne], 12 | 2010-3, mis en ligne le 11 février 2011, Consulté le 21 mai 2012. URL : http://espacepolitique.revues.org/index 1752.html

Engel, H. (2012) : « Les noms de people et de personnages célèbres en traduction », Synergies Pays Scandinaves, $\mathrm{n}^{\circ}$ 7, 43-56.

Fevre-Pernet, C. (2008). « Stratégies dénominatives en onomastique commerciale », in Durand J. Habert B., Laks B. (éds.) Actes du Congrès Mondial de Linguistique Française - CMLF'08, 1539-1550.

- (2011). «Stratégies dénominatives dans la politique de l'eau en France. LEMA, PAPI, SANDRE et les autres », Mots. Les langages $d u$ politique [En ligne], 95 | 2011, consulté le 11 janvier 2015. uRL : http://mots. revues.org/20095.

Giannetti, L. (2002): Understanding movies, NJ Prentice Hall, Upper Saddle River.

Guillot, F. (2004) : « Contrôle et marquage de l'espace : l'appropriation de l'espace frontalier $», E S O \mathrm{n}^{\circ} 21,19-22$.

Hiernaux, P. ex Houerou, H. N. L. (2006) : Les parcours du Sahel, Sécheresse, $17(1-2), 51-71$.

HJelmslev, L. (1971) : Prolégomènes à une théorie du langage, Paris, Les Editions de Minuit.

Holvoet, M. (2013) : Laurent Mauvignier et le minimalisme: l'écriture du non-dit, Mémoire de Maîtrise, Universiteit Gent.

Houli, F. (2006) : « La construction et la politisation de l'ethnicité «kirdi» au nord du Cameroun ", Polis/R.C.S.P./C.P.S.R. vol. 13, ${ }^{\circ} 1$ - 2, pp. 81-102. 
KLEIBER, G. (1984) : « Dénomination et relations dénominatives », Langages, 19e année, $\mathrm{n}^{\circ}$ 76, 77-94.

Kouidri, F. (2009) : « Contact de langue et positionnement identitaire : la langue métissée du rap algérien », Synergies Algérie, n 8, 123-138.

Lallement, M. (2012) : Histoire des idées sociologiques, Tome 2. De Parsons aux contemporains, Armand Colin, Paris.

LeColle, M. ET AL. (dir.) : (2009) : Le nom propre en discours, « Les Carnets $d u$ Cediscor», $n^{\circ}$ 11, Carnets de lecture n. 9, 10, 0, http://farum.it/ lectures/ezine_articles.php?id=135.

Mannheim, K. (1929) : Idéologie et utopie (Une introduction à la sociologie de la connaissance), Macintosh, Chicoutimi.

Mulo Farenkia, B. (2011) : «Formes de “mise à distance" de l'altérité ethnique au Cameroun », Journal of Pragmatics, vol. 43, Issue 6, 1484-1497.

Noumbi WA Kalombo, D. (2009) : « La praxéologie du langage à travers les maximes latines dans la ville de Kananga », SudLangues, ${ }^{\circ}$ 11, pp. 44-57.

Paveau, M.-A. (2008) : " Le toponyme, désignateur souple et organisateur mémoriel. L'exemple du nom de bataille », Mots. Les langages du politique [En ligne], 86 |2008, mis en ligne le 30 mars 2010, consulté le 13 octobre 2012. URL : http://mots.revues.org/13102.

Seignobos, C. \& Tourneux, H. (2002) : Le Nord-Cameroun à travers ses mots. Dictionnaire de termes anciens et modernes, IRD-Karthala, Paris.

TAJE, M. (2010) : "Vulnérabilités et facteurs d'insécurité au sahel », Note publiée par le Secrétariat du Club du sahel et de l'Afrique de l'Ouest (CSAO/OCDE), $\mathrm{n}^{\circ} 1$.

Trepos, J-Y. (2005) : « La sociologie postmoderne est-elle introuvable? », Le Portique [En ligne], 1 | 1998, mis en ligne le 15 mars 2005. URL : http://leportique.revues.org/index346.html.

Vezeanu, I. (2004) : « Moi-même comme un autre. Identité personnelle et langage », in I. Copoeru et N. Szabo (éd.), Beyond Identity. Transformations of Identity in a (Post-) Modern World, Cluj-Romania : Editura Casa Cærflii de Øtiinflæ, 104-124. 
Annexe : Le corpus, distribution géographique et référentielle

\begin{tabular}{|c|c|}
\hline \multicolumn{2}{|c|}{ Garoua (Nord) } \\
\hline Occurrences & Référents \\
\hline Sahel spring & Source d'eau \\
\hline Eil du Sahel & Kiosque journal \\
\hline Sahel shop & Boutique \\
\hline Crédit du sahel & Banque \\
\hline Tempête du sahel & Boite de nuit \\
\hline Kossam sahel & Fabrication yaourt \\
\hline Walde sahel & Cafétariat \\
\hline Water sahel & Vente d'eau \\
\hline Sahel express & Agence de voyage \\
\hline Sahel alimentation & Magasin \\
\hline Sahel couture & Atelier de couture \\
\hline Depot sahel & Boutique \\
\hline Sahel pressing & Pressing \\
\hline Sahel show & Bar \\
\hline Café sahel & Cafetariat \\
\hline Veto sahel & Pharmacie veterinaire \\
\hline Sahel music & Discothèque \\
\hline Coifure du sahel & Salon de coiffure \\
\hline Lavage sahel & Laverie \\
\hline Gic sahel & Vente des bovins \\
\hline Couture sahel & Atelier de couture \\
\hline Alimentation du sahel & Boutique \\
\hline Gic des éleveurs du sahel & Bureau d'un gic \\
\hline Padama sahel & Boutique \\
\hline Gic riba demri du sahel & Gic \\
\hline Restaurant du sahel & Restaurant \\
\hline Discothèque du sahel & Maison de disque \\
\hline Super coiffure le sahel & Salon de coiffure \\
\hline Restaurant du sahel & Restaurant \\
\hline Sahel musique & Discothèque \\
\hline La coiffure du sahel noir de ngal bidje & Salon de coiffure \\
\hline Sahel touristique & Agence \\
\hline Sahel shopping & Boutique \\
\hline
\end{tabular}




\begin{tabular}{|c|c|}
\hline \multicolumn{2}{|c|}{ Maroua (Extrême-nord) } \\
\hline Occurrences & Référents \\
\hline Cabinet vétérinaire du Sahel & Vente des produits. \\
\hline HOTEL le SAHEL & Habitat \\
\hline $\begin{array}{l}\text { Walde Sahel vente boissons hygiénique } \\
\text { Gros détails }\end{array}$ & $\begin{array}{c}\text { Vente de boissons et du lait } \\
\text { naturel }\end{array}$ \\
\hline «Sahel Springs » la meilleure eau minérale & Eau minérale \\
\hline Pharmacie du Sahel & pharmacie \\
\hline Boulangerie du Sahel Call - Box & $\begin{array}{l}\text { Vente du crédit de communi- } \\
\text { cation }\end{array}$ \\
\hline Complexe Sahel clean pressing & Blanchissage \\
\hline Dépôt boulangerie du Sahel vente du pain & Vente du pain \\
\hline Haute coiffure du Sahel & Confection des coiffures \\
\hline La tribune sahélienne presse & Presse \\
\hline Sahel -Boutique & Vente de produits \\
\hline Oasis du sahel & Vente de jus \\
\hline Sahel call Box transfert & TIC \\
\hline Labo sahel photo & Photographie \\
\hline Sahel Springs eau minérale naturelle & Vente eau minérale naturelle \\
\hline Crédit su sahel sA & Finances \\
\hline Agence de voyage Sahel $\mathrm{CH}$ & Transport \\
\hline Sahel espace informatique & TIC \\
\hline Poissonnerie du sahel dépôt général & $\begin{array}{l}\text { Vente et distribution de pois- } \\
\text { son }\end{array}$ \\
\hline PнYтOS du Sahel Gic & Agropastoral \\
\hline Sahel Spring & Vente eau minérale \\
\hline GIC SATOUR Sahel auto location et tourisme. & Location auto et tourisme \\
\hline Sahel coiffure Nas & Coiffure masculine \\
\hline MDC couture sahélienne et occidentale & Couture et vente de tissus \\
\hline Gic le Sahel & Agropastoral \\
\hline Sahel Spring & Vente eau minérale \\
\hline SOURIA-BAI Discothèque - Sahel- Dougoï & Loisir \\
\hline Pâtisserie du Sahel & Pâtisserie \\
\hline La sahélienne & TIC \\
\hline $\begin{array}{l}\text { Institut Supérieur du Sahel / The Higher } \\
\text { Institute of Sahel }\end{array}$ & Enseignement \\
\hline
\end{tabular}




\begin{tabular}{|c|c|}
\hline \multicolumn{2}{|c|}{ Ngaoundéré (Adamaoua) } \\
\hline 1. Occurrences & 2. Référents \\
\hline Gic sahel express & Transport \\
\hline Planète sahel & Restaurant \\
\hline Sahel couture & Salon de couture \\
\hline Boukarou sahel & Restaurant \\
\hline Sahel spring & Eau minérale \\
\hline Restaurant le sahel & Restaurant \\
\hline Oeil du Sahel & Presse \\
\hline Chétima -Sahel & Transport \\
\hline Sahel- photo & Photographie \\
\hline Sahel-info & Secrétariat \\
\hline Minicité le Sahel & Habitat \\
\hline Alimentation le Sahel & Boutique \\
\hline
\end{tabular}

